\title{
MYCOBACTERIUM AVIUM INTRACELLULARE INFECTION OF HIP ARTHROPLASTIES IN AN AIDS PATIENT
}

\author{
JEFFREY R. MCLAUGHLIN, MAUREEN TIERNEY, WILLIAM H. HARRIS
}

Mycobacterium avium intracellulare (MAI) is rarely a human pathogen, but there has been a sharp increase in the incidence of atypical mycobacterial infections in association with AIDS (Hoy et al 1990; Horsburgh 1991), with reports of cardiac, gastrointestinal and respiratory infections (Packer, Cesario and Williams 1988). A literature search revealed no reported case of MAI infection in total hip arthroplasty.

Case report. A 20-year-old man with severe bilateral osteoarthritis of the hips secondary to Perthes' disease had a cemented right replacement with trochanteric osteotomy in 1972 and a similar operation on the left in 1973. By 1980, he had asymptomatic loosening of both components on the left and had a cemented revision.

He became HIV-positive in 1988, and in August 1991 developed Pneumocystis pneumonia and was diagnosed as having AIDS. He was treated with AZT, trimethoprim, sulphamethoxazole and acyclovir and remained well until April 1992, when he developed increasing pain in both hips, only being able to walk less than two blocks even with crutches. He had no skin lesions and his surgical scars remained well healed. Radiographs showed loosening of both acetabular components with surrounding lysis (Fig. 1). There was also some lysis about both femoral components.

In April 1992, hip aspirations revealed Mycobacterium avium intracellulare in both hips and his blood cultures were also positive for MAI. Treatment was with four oral antibiotics: ciprofloxacin $750 \mathrm{mg}$ and clarithromycin $500 \mathrm{mg}$ each twice daily, and rifampicin $600 \mathrm{mg}$ and clofazimine $100 \mathrm{mg}$ each four times daily, in addition to his AZT, trimethoprim and sulphamethoxazole and acyclovir.

In June 1992, at resection arthroplasty of the right hip, the acetabular component was grossly loose but the femoral component was well fixed. There was marked wear of the polyethylene liner. Vancomycin was added to

J. R. McLaughlin, MD, Orthopaedic Surgeon

Kennedy Center for the Hip and Knee, Suite 100, Theda Clark Medical Plaza, Neenah, Wisconsin 54956, USA.

M. Tierney, MD, MSc, Assistant Physician, Division of Infectious Disease W. H. Harris, MD, Chief, Hip and Implant Unit

Orthopaedic Biomechanics Laboratory, Massachusetts General Hospital, 32 Fruit Street, Boston, Massachusetts 02114, USA.

Correspondence to $\mathrm{Dr}$ W. H. Harris.

(C)1994 British Editorial Society of Bone and Joint Surgery 0301-620X/94/3R05 $\$ 2.00$

J Bone Joint Surg [Br] 1994; 76-B:498-9.

Received 9 July 1993; Accepted 31 August 1993 his drugs after aerobic, anaerobic, mycobacterial and fungal cultures had been obtained, and a Hickman catheter placed.

Histological examination of operative specimens showed clusters of acid-fast bacilli in necrotic tissue with minimal inflammatory response (Fig. 2). The specimens grew MAI and a very rare coagulase-negative staphylococcus on one culture, and one colony only of staphylococcus on another. Because of pyrexia after operation, ethambutol was added to his antibiotics and the dose of clarithromycin was increased to $1 \mathrm{~g}$ twice daily.

Bed rest with the involved leg in balanced suspension continued for six weeks, after which the Hickman catheter was removed and he was mobilised on two crutches.

The patient was discharged to his home 45 days after admission, on nine oral antimicrobial drugs, four AIDS drugs and warfarin for a deep femoral vein thrombosis detected by ultrasound. He died five months later.

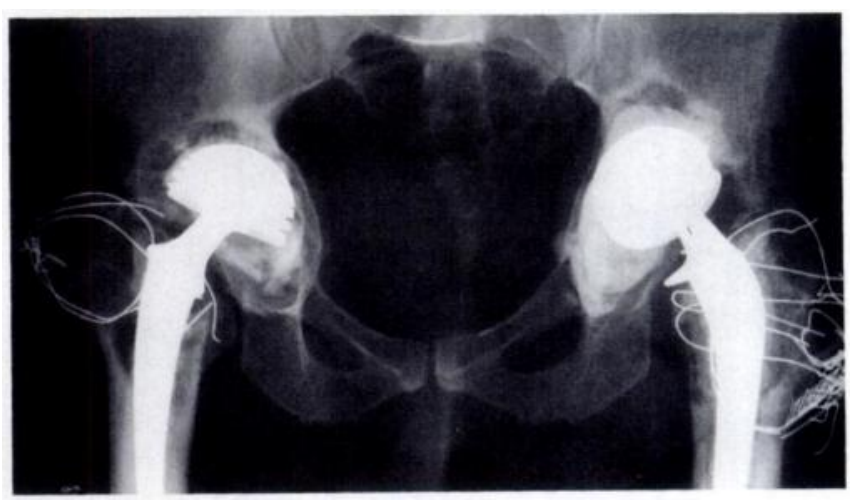

Fig. 1

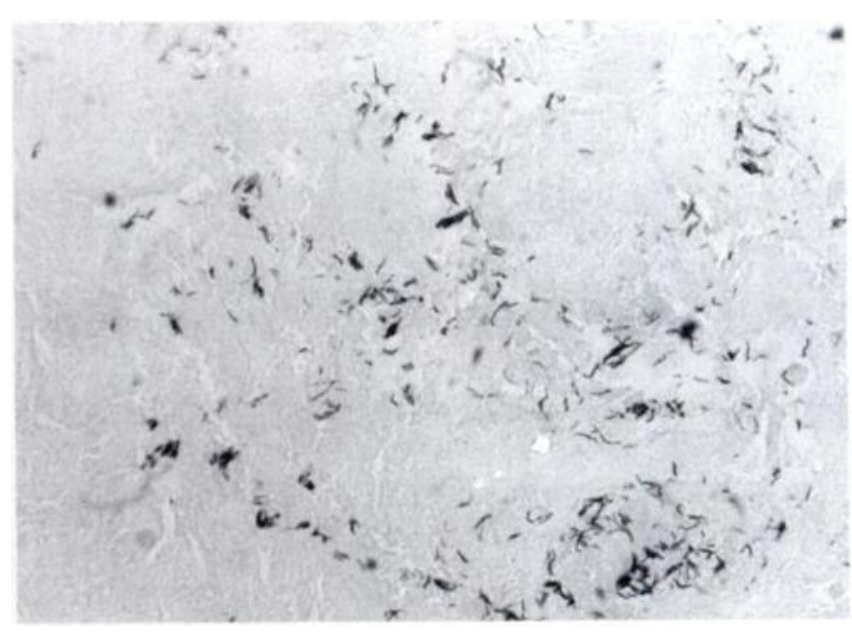

Fig. 2 
Discussion. Mycobacterium avium intracellulare is a ubiquitous organism found in soil, food and water (Ellner, Goldberger and Parenti 1991). MAI infections are rare and usually occur in elderly patients with chronic lung disease or patients who are immunocompromised. Before 1980 only 24 cases of disseminated MAI infections had been reported (Horsburgh 1991), but recently there has been a marked increase in both Mycobacterium tuberculosis and atypical mycobacterial infections in AIDS patients. MAI infections are reported to occur in $15 \%$ to $24 \%$ of patients with AIDS (Hawkins et al 1985; Bessesen et al 1990; Hoy et al 1990) and 53\% of AIDS patients who died at the Memorial Sloan Kettering Cancer Center were found at post-mortem to have disseminated MAI infection (Hawkins et al 1985); similar findings have been reported by Wallace and Hannah (1988).

MAI can usually be isolated and identified using media generally available in clinical microbiology laboratories. The acid-fast bacilli are readily seen on ZiehlNielsen (Horsburgh 1991) or auramine O fluorochrome (Hawkins et al 1985) stains. Cultures of blood, urine, sputum and stool are generally inoculated on to Middlebrook 7H11 agar or Loewenstein-Jensen media (Hawkins et al 1985). Colonies grow in 7 to 51 days (Macher et al 1983; Pierce, De Young and Roberts 1983). Histological examination shows numerous acid-fast bacilli within distended histiocytes. Granulomas are poorly formed and the host inflammatory response is minimal (Horsburgh 1991).

Infections with MAI are characterised by host colonisation predominantly through the respiratory or gastrointestinal tracts, which is followed by haematogenous dissemination. This contrasts with Mycobacterium tuberculosis infections, in which dissemination usually occurs after the reactivation of quiescent disease (Horsburgh 1991).

Disseminated MAI infection causes fever, malaise, weight loss and anaemia, while abdominal pain and diarrhoea are common. Pneumonia is less frequent but may occur (Hawkins et al 1985; Horsburgh 1991). Most organ systems may be involved, however, and musculoskeletal infections involving the wrist, distal ulna, flexor tendon sheath, and knee have been reported (Pedersen, Hald and Saxegaard 1988; Rolfe and Sowa 1990; Stark 1990; Whitaker et al 1991).

In one series, blood cultures were positive in $98 \%$ of patients with MAI, with cultures of lymph nodes and bone-marrow aspirates positive in $100 \%$ (Hawkins et al 1985).

Until recently antibiotics for MAI infection in AIDS had little success, but more aggressive multiple drug regimens have given more promising results. Hoy et al (1990) reported decreased symptoms in 18 of 25 patients, with clearing of bacteraemia in 22 after the use of ethambutol, rifabutin, clofazimine and isoniazid. Chiu et al (1990) had similar success with ethambutol, rifampicin, ciprofloxacin and amikacin. The new macrolide anti- biotics, clarithromycin and azithromycin (Young et al 1991; de Lalla et al 1992), have impressive in vitro activity against MAI, and the use of these drugs in combination with other agents has had promising results (Young et al 1991; de Lalla et al 1992). At present the recommended therapy is four drugs, usually including rifampicin or rifabutin, a new macrolide, and ciprofloxacin.

Neither the effectiveness nor the duration of antibiotic treatment for disseminated MAI infection has been established. We consider that a total joint arthroplasty infected with MAI in a patient with AIDS should be removed and that a prolonged course of antibiotics should be given. In patients with AIDS, MAI infections produce persistent bacteraemia, and reimplantation of an arthroplasty therefore carries an increased risk. Even if infection can be cleared, reconstruction is probably not indicated in patients severely debilitated by AIDS.

No benefits in any form have been received or will be received from a commercial party related directly or indirectly to the subject of this article.

\section{REFERENCES}

Bessesen MT, Berry CD, Johnson MA, et al. Site of origin of disseminated MAC infection in AIDS. Abstracts of the 1990 ICAAC, 1990:297.

Chiu J, Nussbaum J, Bozzette $S$, et al. Treatment of disseminated mycobacterium avium complex infection in AIDS with Amikacin, Ethambutol, Rifampin and Ciprofloxacin. Ann Intern Med 1990; 113:358-61.

Ellner JJ, Goldberger MJ, Parenti DM. Mycobacterium avium infection and AIDS: a therapeutic dilemma in rapid evolution. J Infect Dis 1991; 163:1326-35.

Hawkins CC, Gold JW, Whimbey E, et al. Mycobacterium avium complex infections in patients with the acquired immunodeficiency syndrome. Ann Intern Med 1985; 105:184-8.

Horsburgh CR Jr. Mycobacterium avium complex infection in the acquired immunodeficiency syndrome. New Engl J Med 1991; 324:1332-8.

Hoy J, Mijch A, Sandland M, et al. Quadruple-drug therapy for mycobacterium avium-intracellulare bacteremia in AIDS patients. $J$ Infect Dis 1990; 161:801-5.

de Lalla F, Maserati R, Scarpellini P, et al. Clarithromycin-ciprofloxacinamikacin for therapy of mycobacterium avium-mycobacterium intracellulare bacteremia in patients with AIDS. Antimicrob Agents Chemother 1992; 36:1567-9.

Macher AM, Kovacs JA, Gill V, et al. Bacteremia due to mycobacterium avium-intracellulare in the acquired immunodeficiency syndrome. Ann Intern Med 1983; 99:782-5.

Packer SJ, Cesario T, Williams JH Jr. Mycobacterium avium complex infection presenting as endobronchial lesions in immunosuppressed patients. Ann Intern Med 1988; 109:389-93,198.

Pedersen AK, Hald J, Saxegaard F. Mycobacterium avium infection of the knee in a child. Acta Orthop Scand 1988; 59:585-6.

Pierce PF, De Young DR, Roberts GD. Mycobacteremia and the new blood culture systems. Ann Intern Med 1983; 99:786-9.

Rolfe B, Sowa DT. Mixed gonococcal and mycobacterial sepsis of the wrist. Clin Orthop 1990; 257:100-3.

Stark RH. Mycobacterium avium complex tenosynovitis of the index finger. Orthop Rev 1990; 19:345-8.

Wallace JM, Hannah JB. Mycobacterium avium complex infection in patients with the acquired immunodeficiency syndrome: a clinicopathologic study. Chest 1988; 93:926-32.

Whitaker MD, Jelinek JS, Kransdorf MJ, Moser RP Jr, Brower AC. Case report 653. Skeletal Radiol 1991; 20:291-3.

Young LS, Wiviott $\mathrm{L}, \mathbf{W u ~ M}$, et al. Azithromycin for treatment of Mycobacterium avium-intracellulare complex infection in patients with AIDS. Lancet 1991; 338:1107-9. 